\title{
Kernos
}

Revue internationale et pluridisciplinaire de religion grecque antique

$26 \mid 2013$

Varia

\section{Oracles et mentalités grecques}

La confirmation d'un oracle par une seconde consultation au même sanctuaire

\section{Pierre Bonnechere}

\section{(2) OpenEdition}

Journals

Édition électronique

URL : http://journals.openedition.org/kernos/2202

DOI : 10.4000/kernos.2202

ISSN : 2034-7871

\section{Éditeur}

Centre international d'étude de la religion grecque antique

\section{Édition imprimée}

Date de publication : 10 octobre 2013

Pagination : 73-94

ISSN : 0776-3824

\section{Référence électronique}

Pierre Bonnechere, «Oracles et mentalités grecques », Kernos [En ligne], 26 | 2013, mis en ligne le 31 octobre 2015, consulté le 02 mars 2021. URL : http://journals.openedition.org/kernos/2202 ; DOI : https://doi.org/10.4000/kernos.2202 


\title{
Oracles et mentalités grecques : la confirmation d'un oracle par une seconde consultation au même sanctuaire*
}

\begin{abstract}
Résumé : Manipulés par les puissances hégémoniques, les sanctuaires oraculaires auraient rendu des oracles intéressés, et du même coup ambigus pour laisser aux consultants toute la responsabilité de leurs erreurs d'interprétation. Un coup d'œil aux réponses conservées dans les sources contemporaines des faits (épigraphie et sources littéraires) suffit pour se convaincre du contraire. De plus, il existe une tradition méconnue qui contredit le topos de l'ambiguité volontaire : la seconde consultation du même sanctuaire pour préciser un oracle rendu. On trouve d'abondants exemples, privés et publics, fictifs et réels, sur tous les sujets possibles, de l'archaïsme à la période romaine, à Delphes, Didymes et Dodone, entre autres. Qui plus est, et même dans les cas légendaires ou rapportés longtemps après les faits, la seconde consultation n'est presque jamais la conséquence de l'ambiguïté de la première réponse.
\end{abstract}

Abstract: It is often argued that Greek oracular sanctuaries provided self-interested answers, and that they were manipulated by hegemonic States. Hence, those oracles would have been ambiguous in the responses they gave and would have left the responsibility for any wrong interpretation to the person consulting them. In fact, a quick look at the preserved oracular responses reported by contemporary sources (both in inscriptions and in the literature) proves the opposite. Furthermore, there is a little-known tradition, which contradicts the topos of intentional ambiguity, i.e. a second consultation in the same sanctuary in order to clarify the first response given by an oracle. Many examples, private and public, fictitious and real, on all possible topics, from the archaic to the Roman period, exist at Delphi, Didyma and Dodona, among other places. Even in the legendary cases, or in those reported long after the fact, the second consultation is almost never prompted by the ambiguity of the first answer.

La divination grecque est un étrange champ d'études : d'un côté les historiens intransigeants qui se fondent d'abord sur les inscriptions, de l'autre les historiens plus accommodants, qui accordent un certain crédit aux oracles chez Hérodote. Un autre clivage sépare les historiens de la religion, plus proches du sentiment religieux, et les historiens de la politique, toujours prompts à évacuer cet aspect pour trouver dans les mots du dieu un argument qui renforce leur théorie sur l'hégémonie de Sparte ou les machiavéliques machinations de Thèbes. Chaque

* Cet article est publié grâce à une subvention de recherche du Conseil de recherches en sciences humaines du Canada (CRSH, Ottawa), que je remercie de son soutien. 
groupe a longtemps campé sur ses positions mais, depuis que le corpus de Dodone est mieux connu, l'équilibre a changé, même si peu de gens encore l'ont remarqué1. En fonction de sa tradition littéraire, semblable à celle de Delphes, Dodone présente le pur profil d'un sanctuaire consulté avant tout par les États, pour des raisons de grande politique et, au premier chef, pour la guerre. Cette image toute littéraire se trouve sans appel contredite par l'impressionnante collection des lamelles de plomb aujourd'hui publiée, qui nous a transmis l'original de presque 200 questions à l'authenticité assurée ${ }^{2}$. Sur 191 questions, seules 19 sont publiques $^{3}$, et leur formulation est soit d'un vague consommé («qui prier pour gouverner au mieux ? $»^{4}$ ), soit d'une ponctualité remarquable (" est-il avantageux pour la déesse Thémis de prêter telle somme d'argent ? $»^{5}$ ). Mais une seule concerne la grande politique (en fait une question sur l'opportunité d'une alliance ${ }^{\natural}$ ), et encore vers 170 av. J.-C., pour une époque dont tout le monde affirme, étrangement, que l'influence politique des oracles avait disparu 7 . Aucune ne concerne la guerre. Il reste 4000 tablettes toujours inédites, mais les rares privilégiés à les avoir vues confirment le ratio général, privé à $90 \%$ et, pour autant qu'on puisse le savoir, dans les 10\% restants, l'absence de grande politique ${ }^{8}$. L'inertie, toutefois, est dotée d'une force incroyable. Quand j'ai exposé ces idées à un collègue, il m'a répondu : «oui, mais Dodone est un cas à part», ce qui permet de conserver intacte l'image d'une Delphes idéalisée qui un jour a régenté le monde grec par ses avis tantôt éclairés tantôt corrompus.

${ }^{1}$ Voir surtout É. LHÔTE, Les lamelles oraculaires de Dodone, Genève, 2006 et E. EIDINOW, Oracles, Curses, and Risk among the Ancient Greeks, Oxford, 2007. La thèse de M. DiETERLE (Dodona. Religionsgeschichtliche und historische Untersuchungen zur Enstebung und Entwicklung des Zeus-Heiligtum, Hildesheim/New York, 2007 [Spudasmata, 116]) est une remarquable synthèse sur le site et les fouilles, mais elle apporte peu de neuf sur les inscriptions et le rapport des Grecs à l'oracle de Zeus.

${ }^{2}$ Sur l'oracle de Dodone, ses soi-disant implications politiques et sa tradition littéraire, voir P. BONNECHERE, «Los oráculos griegos y la gran política. Un contra-ejemplo: el oráculo de Dodona y la 'Guerra de las lágrimas' en Eutresis en 368/67 a.C.», in M. CAMPAGNO, J. Gallego, C. García McGaw (éd.), Política y religión en el Mediterráneo antiguo, Buenos Aires, 2009, p. 273-286 (une version plus étoffée est en cours de réalisation).

${ }^{3}$ Voir LhÔTE, o.c. (n. 1), nos 1-5, 6A, 7, 8B, 9-17 \& 137 et EidinOw, o.c. (n. 1), p. 346, n 6.

${ }^{4}$ LHÔTE, o.c. (n. 1), nos 1-2, 6A (cf. aussi no 7 ).

${ }^{5}$ LHÔTE, o.c. (n. 1), no 137.

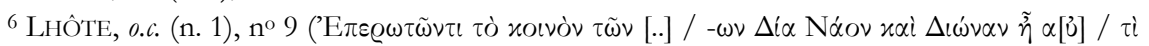

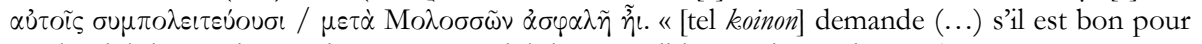
sa sécurité de conclure maintenant un traité de sympolitie avec les Molosses »).

7 Voir par exemple R. PARKER, "Greek States and Greek Oracles », in R. BUXTON (éd.), Oxford Readings in Greek Religion, Oxford, 2000 [1985], p. 76-108, part. 87-88. Parker est suivi par presque tous les historiens sur ce sujet : par exemple H. BOwDEN, Classical Athens and the Delphic Oracle. Divination and Democracy, Cambridge, 2005, p. 114-117, 132-151 (travail au demeurant excellent). Voir cependant mon article «The Religious Management of the Polis: Oracles and Political Decision-Making ", in H. BECK (éd.), A Companion to the Greek Governement, Malden, et al., 2013, p. 366-381.

${ }^{8}$ R. PARKER, communication personnelle. 
En fait, l'étude de la divination grecque est viciée par les multiples globalisations qu'elle a subies, et ce dès l'antiquité. Ces globalisations amalgament les divers types de divination telle qu'elle fut pratiquée et l'image multiforme que les anciens se faisaient de la mantique. Aucun texte oraculaire transmis par l'épigraphie n'est en vers avant le II siècle av. J.-C. (et encore ${ }^{9}$ ), quand la plupart de ceux de la tradition littéraire ancienne le sont. Pour pallier la contradiction, un auteur comme Plutarque déjà, dans ses Dialogues pythiques, sera astreint à de multiples contorsions intellectuelles, pour ne rien dire des nôtres. C'est l'une de ces généralisations qui a aiguillé le propos de cet article : l'ambiguiité des oracles ${ }^{10}$.

Dans l'imaginaire hellénique en effet, et par voie de conséquence aussi dans les travaux modernes, les questions posées aux oracles, souvent en temps de crise, sont ouvertes, du genre "Que devons-nous faire ? ", et les réponses s'en trouvent dangereuses pour les particuliers et les cités : "Allez coloniser Cyrène » ou «Rappelez tous les bannis » ${ }^{11}$. C'est que les «prêtres»y dicteraient leur vouloir comme bon leur semblerait, à la botte ou non des puissances dominantes. Pour se garder d'éventuelles erreurs, de surcroît, lesdits oracles répondraient de façon ambiguë. Toute l'interprétation et toute erreur potentielle auraient donc pesé sur les épaules du consultant. À la différence des prophéties consignées longtemps après la consultation, toutefois, les oracles relatés dans l'épigraphie, ou dans les sources littéraires contemporaines des faits, concernent toujours des questions extrêmement ponctuelles, codifiées en quelque sorte, et leurs réponses, elles aussi codifiées, ne sont jamais ambiguës avant l'époque romaine : «est-il meilleur et plus avantageux pour lui de demander à la cité un endroit qu'il a en tête, dans le sanctuaire d'Apollon (...), où il pourra élever un temple d'Aphrodite ? ». Réponse de Delphes : «le dieu lui a répondu de demander dans le temple d'Apollon ${ }^{12}$.

9 J. Fontenrose, The Delphic Oracle. Its Responses and Operations, with a Catalogue of Responses, Berkeley et al., 1978, H 56 (inscription de Pharos) : IG XII suppl., 200. Restitutions d'un hexamètre par L. RoBERT, BCH 59 (1935), ad 1. 24-26. Voir cependant G. DAux apud P. AmANDRY, La mantique apollinienne à Delphes, Paris, 1950, p. 155, n. 2, sceptique sur les lectures proposées d'un texte très usé, et le point par P. AMANDRY, « Propos sur l'oracle de Delphes », JS (1997), p. 205, n. 33 (aporétique).

${ }^{10} \mathrm{La}$ recherche, là aussi, est surprenante. On sait depuis longtemps que l'ambiguïté n'est pas une caractéristique des oracles grecs (par ex. J. Fontenrose, Compte rendu de H.W. PARKE, History of the Delphic Oracle [Oxford, 1939], AJPh 63 [1942], p. 475; AMANDRY, o.c. [n. 9], M. DelcourT, L'oracle de Delphes, Paris, 1955, p. 85-108 ou R. CRAHAY, La littérature oraculaire chez. Hérodote, Paris, 1956, p. 6-11, 343-344), avis martelé encore par FonTENROSE (o.c. [n. 9], passim), mais c'est à croire que tous ont prêché dans le désert, ce qui rend encore et toujours nécessaires de nouvelles mises au point, comme celles de BowDEN, o.c. (n. 7) et G. ROUGEMONT, «Les oracles grecs recouraient-ils habituellement à l'ambiguïté volontaire? », in L. BASSET, F. BIVILLE (éd.), Les jeux et les ruses de l'ambiguité volontaire dans les textes grecs et latins, Lyon, 2005, p. 219-235.

11 Cyrène : Fontenrose, o.c. (n. 9), Q 45-48 (= Hérodote, IV, 150-156). Rappel des bannis : Fontenrose, o.c. (n. 9), Q 170 (= Pausanias, VI, 11, 7; Oinomaos apud Eusèbe, Préparation évangélique $\mathrm{V}, 34)$.

12 Fontenrose, o.c. (n. 9), H 54 (IG XIII 3, 248, 1. 29-32) : sanctuaire d'Anaphé, vers 100 av. J.-C. 
«Cléôtas demande à Zeus et à Diona s'il est avantageux et profitable pour lui de se consacrer à l'élevage des moutons?»On ignore si Zeus répondit oui ou non, mais le Cléôtas en question dut certainement trouver la réponse de Dodone éclairante ${ }^{13}$. Or, si les questions évitent les sujets interdits à la raison humaine, et si les réponses sont claires et focalisées à l'extrême, c'est un des poncifs de l'étude de la divination qui s'en trouve malmené, à savoir l'interprétation des oracles. Toutes les belles histoires d'interprétation maladroite, à double entendre, tombent dans la catégorie des oracles rapportés longtemps après les faits ${ }^{14}$, la plus célèbre étant l'oracle du mur de bois interprété par Thémistocle ${ }^{15}$. Non que l'interprétation n'ait jamais été requise : un signe fortuit, un rêve, peuvent nécessiter le recours à l'interprète spécialisé16. De façon paradoxale même, les visions extatiques obtenues de Trophonios qui, pour les philosophes, trônent au sommet de l'art divinatoire grâce au contact direct entre l'âme et le dieu, exigent des prêtres qu'ils fouillent dans la mémoire du consultant pour comprendre ce qu'il a « vu et entendu $»^{17}$. Mais l'interprétation n'a jamais eu le poids écrasant que la littérature, et la recherche à sa remorque, lui ont donné, car les anciens l'ont limitée au maximum en travaillant les modes d'interrogation et de réponse : questions claires et ponctuelles, réponses claires et précises ${ }^{18}$.

Qui plus est, il est une habitude assez répandue, passée inaperçue jusqu'à présent, qui contredit l'idée même de l'interprétation, personnelle ou publique, rendue nécessaire par l'oracle ambigu : il s'agit de la double interrogation d'un même manteion, parfois à très bref intervalle, pour préciser l'oracle rendu. La littérature de fiction livre son comptant d'exemples, mais les inscriptions ne sont pas en reste, du VI siècle av. J.-C. à l'époque romaine. Le tableau cidessous donne quelques chiffres généraux: les cas attestés par des sources contemporaines des faits, - essentiellement épigraphiques, - sont au nombre de dix-huit contre trente-deux dans le domaine des consultations imaginaires ${ }^{19}$.

${ }^{13}$ LHÔte, o.c. (n. 1), no 80 : Dodone, ve s. av. J.-C.

${ }^{14} \mathrm{La}$ fameuse catégorie « quasi-historical » de Joseph Fontenrose. Voir infra, n. 33.

${ }^{15}$ FonTENROSE, o.c. (n. 9), Q 147 (voir infra).

16 Par exemple : Théophraste, Caractères, 16, 6 et 11.

17 Pausanias, IX, 39, 13. C'est paradoxal car on lit très souvent, y compris sous mon nom, que les oracles qui se passaient d'intermédiaire avaient mieux résisté au temps. Raisonnement exact mais inachevé : cela signifie que l'interprétation y était secondaire par rapport au contact direct avec le dieu. Nous retrouvons la primauté du pathein sur le mathein, dont on peut mesurer l'ampleur dans la consultation de Trophonios. Voir P. BonneChere, Trophonios de Lébadée. Cultes et mythes d'une cité béotienne au miroir de la mentalité grecque, Leyde et al., 2003, part. p. 139-202.

${ }^{18} \mathrm{Il}$ arrive très souvent que la réponse de l'oracle ne soit qu'une des deux possibilités d'une alternative présentée à la divinité : BONNECHERE, l.c. (n. 7), p. 375-376.

${ }^{19}$ Je n'ai pas fait le décompte pour les sanctuaires médicaux, faute d'un catalogue fiable à l'heure actuelle. 


\begin{tabular}{|c|c|c|c|}
\hline Oracle & $\begin{array}{l}\text { Textes contempo- } \\
\text { rains des faits } \\
\text { (= historiques })\end{array}$ & $\begin{array}{c}\text { Textes postérieurs } \\
\text { aux faits (= quasi- } \\
\text { historiques) }\end{array}$ & $\begin{array}{c}\text { Récits clairement } \\
\text { légendaires }\end{array}$ \\
\hline Claros (Apollon) & $\mathrm{H} 0 / 34$ & Q 0/11 & $\mathrm{L} 0 / 0$ \\
\hline Delphes (Apollon) ${ }^{20}$ & $\mathrm{H} 3 / 75^{21}$ & Q $19 / 251^{22}$ & L $12 / 171^{23}$ \\
\hline Didymes (Apollon) & H 5 ou $6 / 3924$ & Q $1 / 25^{25}$ & $\mathrm{~L} 0 / 4$ \\
\hline Dodone (Zeus) & $\begin{array}{l}\text { H 0/7 (littérature) } \\
\text { H 5/188 (lamelles) }{ }^{26}\end{array}$ & Q 0/17 & L 0/16 \\
\hline Lébadée (Trophonios) & H 4/13 (min.) $)^{27}$ & Q 0/10 & $\mathrm{L} 0 / 6$ \\
\hline Olympie (Zeus) & $\mathrm{H} 0 / 7$ & Q $0 / 0$ & $\mathrm{~L} 0 / 0$ \\
\hline Siwa (Ammon) & $\mathrm{H} 0 / 2$ & Q 0/17 & $\mathrm{L} 0 / 6$ \\
\hline
\end{tabular}

Il est impossible de produire des statistiques exactes, car ces données sont, tout au plus, minimales : nous ne savons si une question a déjà été posée au dieu

\footnotetext{
${ }^{20}$ Fontenrose catalogue $268 \mathrm{Q}$ (uasi-historiques) et 176 L(égendaires). J'ai déduit des totaux le nombre de $\mathrm{Q}$ et $\mathrm{L}$ qui sont des consultations doubles (ou multiples) pour ne conserver que le nombre de consultations (et non le nombre de réponses oraculaires).

${ }^{21}$ Fontenrose, o.c. (n. 9), H 11 et 74, auxquels j'ajoute Xénophon, Revenus, 6.

${ }^{22}$ Fontenrose, o.c. (n. 9), Q $\{8-9\},\{28-30\}, 31,\{34-35\},\{47,49,51\}, 63,\{89-90\}$, $\{99-100$, $103\},\{104-105\}, 140,\{146-147\}, 164,\{170-171\}, 191,250,\{253-256\}, 268$ (source médiévale). À ces 16 cas, j'ajoute Q 232 (qui comporte un refus de réponse de la part d'Apollon, suivi de la réponse suite à une nouvelle interrogation, dans le cas des vierges locriennes) et $\mathrm{Q}\{109-110\}$, où des consultants différents interrogent l'oracle mais sur un même problème.

${ }^{23}$ Fontenrose, o.c. (n. 9), L 6, 42, 63, 64, \{68-70\}, \{92-93\}, 162, 163-167, 173-174. À ces neuf cas, j'en ajoute trois : L 109 comporte un refus de la part d'Apollon, suivi de la réponse suite à une nouvelle interrogation. L 153 regroupe deux consultations faites par des personnes différentes le même jour et dont les réponses sont entrelacées. L $\{72-73\}$ illustre des consultations multiples, mais un cadre moins net: alors que les Argiens s'inquiètent de la chaleur engendrée par Sirius, Pytho leur enjoint d'apaiser Psamathe et Linos, ou encore de sacrifier au monstre, Poiné-Kèr, le jeune homme qui l'a tué. Coroibos, ayant eu vent de l'oracle, se rend à Delphes et demande au dieu de disposer de sa vie. Apollon lui ordonne de ne pas rentrer à Argos mais de partir avec un trépied sur les épaules pour fonder un temple à l'endroit où il devrait le déposer : entre autres Anthologie palatine VII, 154; Callimaque, Aitia, fr. 26-31 (Pfeiffer); Conon, FrGrHist., 26 F 1 (19); Pausanias, I, 43, 7-8; Stace, Thébaïde I, 638-668.
}

24 J. Fontenrose, Didyma. Apollo's Oracle, Cult, and Companions, Berkeley et al., 1988, (H) 1 , 24, 29, 31; A 4; peut-être (H) $\{22-23\}$.

\footnotetext{
${ }^{25}$ FONTENROSE, o.c. (n. 9), (H) $\{38-39\}$.

${ }^{26}$ LHÔTE, o.c. (n. 1), nos 10Ab, 95AB, 107A, 131, 135

27 BONNECHERE, o.c. (n. 17), p. 59-60. IG VII, 3055.
} 
sur le même sujet que si le texte en fait mention, explicitement ou non ${ }^{28}$. En plusieurs cas, c'est une nuance, ou une tournure, ou encore un terme qui autrement serait superflu qui trahit la nouvelle question ${ }^{29}$. Si cette pratique n'est pas fréquente, elle est quand même relativement bien établie, mieux documentée encore pour la consultation successive de deux sanctuaires différents sur une même question, dont je ne citerai ici qu'un exemple ${ }^{30}$. En 368 av. J.-C., le régent de Sparte Agésipolis est en guerre contre les Argiens, qui s'abritent aussitôt derrière la trêve sacrée des Carneia - une fête pan-dorienne qui interdit toute action militaire. Comme la période de l'année n'était pas celle des Carneia ${ }^{31}$, Agésipolis interroge l'oracle de Zeus à Olympie, qui l'assure de ce qu'une trêve indue n'est pas valide. L'oracle de Delphes, interrogé juste après, lui répond de même. La théorie traditionnelle, quand elle en parle, invoque une tentative de contrôler le risque d'erreur, ou une défiance face aux fameuses réponses abusives ou ambiguës des prêtres et autres pythies. H. Parke et D. Wormell, et d'autres modernes à leur suite ${ }^{32}$, voient dans la conduite d'Agésipolis le signe du rationalisme croissant des élites qui déprécient désormais la divination, et une façon sophistique d'» extorquer » d'Apollon la confirmation de l'oracle de son père Zeus d'Olympie, un oracle qu'il n'aurait pu contredire. Il faut toutefois, pour défendre cette position, réduire les Grecs à des athées au sens actuel du terme, feindre d'oublier l'attachement de tous les philosophes grecs, ou presque, à la divination, méconnaître Xénophon dont l'Agésipolis est un modèle de piété et de respect des volontés divines, et enfin passer sous silence la tradition de double consultation que je viens d'évoquer. Aussi la fameuse «manipulation» ne doit-elle pas plutôt être perçue comme une façon pieuse d'approcher la divinité dans cet exercice extrêmement périlleux qui consiste à choisir dans le présent l'orientation que prendra le futur, et sans hypothéquer la protection divine indispensable à l'harmonie civique? Rompre une trêve sacrée, même douteuse, sans l'approbation divine, serait faire preuve d'inconscience politique.

\footnotetext{
${ }^{28}$ Beau cas dans l'anagraphe de Lindos (D 95-115). Voir C. HigBIE, The Lindian Chronicle and the Greek Creation of Their Past, Oxford, 2003, p. 149-151. Un ancien prêtre d'Athéna Lindia, nommé Calliclès, se voit ordonner par la déesse, et six nuits de suite, de persuader les autorités à tenter de rallier Ptolémée Ier à leur cause, pendant le siège de Démétrios Poliorcète. Nous ignorons les modalités pratiques de l'histoire, sinon que l'épiphanie divine en rêve est, apparemment, spontanée et non recherchée.

${ }^{29}$ Par exemple infra, 2.1.8 ou 2.2.1.

30 Je renvoie à mon article, «Oracles and Greek Mentalities: The Mantic Confirmations of Mantic Revelations », in J. Dijkstra, J. Kroesen, Y. Kuiper (éd.), Myths, Martyrs, and Modernity. Studies in the History of Religions in Honour of Jan N. Bremmer, Leyde et al., 2010, p. 115-133.

${ }^{31}$ Xénophon, Helléniques IV, 7, 1-3.

32 The Delphic Oracle II, Oxford, 1956, p. 209-210 («it shows how weak a formality the consultation of the oracle was, if Agesipolis felt no compunction in forcing a favourable reply by such a transparent sophistry »).
} 


\section{Dans la littérature consignée longtemps après les faits et dans les récits légendaires}

Force est de constater que la seconde question destinée à préciser la première est bien représentée dans l'imaginaire grec sur les oracles. Il existe même des exemples de consultations multiples, dont l'une va jusqu'à cinq questions consécutives. On ne s'appesantira pas ici sur la lancinante question de l'authenticité des prophéties rendues. Je me borne à reprendre la classification de Joseph Fontenrose, qui s'impose par sa logique : face aux enquêtes légendaires qui, fictives, peuvent néanmoins refléter des usages réels, on distingue les consultations selon qu'elles ont été rapportées par des sources contemporaines des faits (historiques) ou non (quasi-historiques) ${ }^{33}$.

L'interrogation multiple est parfois solidaire du topos de la mauvaise interprétation. Dans le domaine légendaire, le roi héraclide Téménos, après ses pères, veut ainsi reconquérir le Péloponnèse. Il reçoit, à Delphes, les mêmes réponses qu'Apollon a faites à ses devanciers, et réprimande la pythie : ces informations, pourtant respectées par les rois n'ont-elles pas mené à autant d'expéditions désastreuses? Dans son dialogue avec la pythie sur les oracles «mensongers» du dieu, dont ceux des «trois moissons » et des «chemins étroits ", Téménos demande une précision qu'il finit par obtenir, et qui lui ouvre la voie du succès ${ }^{34}$. Dans le Prométhée enchaîné, Inachos tente en vain de percer les rêves atroces de sa fille, et dépêche maints messagers à Delphes et Dodone, qui reviennent porteurs de messages ambigus, jusqu'à ce qu'enfin la clarté se fasse : il doit chasser Io sous peine de voir Zeus détruire toute sa maison ${ }^{35}$. Enfin, les fils du roi Codros, décédé lors d'une invasion spartiate, se disputent le pouvoir. La pythie leur avait dit qu'aurait la royauté celui qui le premier ferait une libation après avoir vu un sialos se frotter contre un sialos. Or sialos a deux acceptions : " cochon » et « olivier». Médon l'emporte sur Neileus, qui néanmoins contrôle à Delphes l'interprétation de son frère. La pythie lui confirme que sialos signifie bien « olivier ». Neileus veut alors savoir dans quelle cité il devra s'installer, et est sommé de fonder une colonie ${ }^{36}$.

Ne concluons pas trop vite que l'ambiguïté et la faille dans l'interprétation commandaient toute nouvelle interrogation, au contraire. Seul le premier cas contient une réelle ambiguïté, car dans l'histoire d'Inachos un oracle clair est

${ }^{33}$ FonTENROSE, o.c. (n. 9), p. 8, et surtout p. 42-57. En dépit de certains raccourcis, dus à une volonté de classification trop raide pour une matière trop fluide, l'analyse de Fontenrose demeure inégalée dans sa qualité méthodologique. Elle vint trop tôt dans le monde universitaire, qui n'était pas préparé, dans les années 1970, à abattre un de ses dogmes, l'interventionnisme delphique.

34 Fontenrose, o.c. (n. 9), L 63 : résumé dans Apollodore, 2, 8 (cf. aussi L 62 \& 65).

35 Fontenrose, o.c. (n. 9), L 6 : Eschyle, Prométhée enchaîné, 645-671.

36 Fontenrose, o.c. (n. 9), L \{68-70\} : Élien, Histoires variées, 8, 5; Pausanias, VII, 2, 1. Oracle conservé par le scholiaste d'Aelius Aristide, 13, 110 (p. 78 Dindorf) et Tzétzès ad Lycophron, Alexandra, 1378 (cf. Chiliades, 13, 101-103). 
finalement délivré, et dans celle des fils de Codros, il s'agit d'un double entendre qu'un des frères parvient à déchiffrer. Neileus en profite par ailleurs pour demander très clairement quoi faire suite à l'intronisation de Médon. De plus, tous les autres exemples se signalent par leur clarté : ainsi les Orchoméniens, terrassés par un fléau, se voient enjoindre de ramener de Naupacte les os d'Hésiode. Aussitôt ils veulent apprendre comment trouver la dépouille du poète : un corbeau, dit la pythie, leur montrera ${ }^{37}$. Durant son dialogue avec le dieu sur le retour des Héraclides, le roi Téménos interroge la prophétesse sur un fléau, dont la cause, apprend-il, est le meurtre de Carnos. Il s'enquiert alors de comment propitier Apollon, ce à quoi on lui répond d'instaurer les Carneia $a^{38}$. Deux générations après Oreste, Comètès demande à Apollon où mener sa colonie. Par trois fois, il n'obtient pas de réponse, puis se voit enjoindre de mettre le cap au plus profond de la Mysie. Comètès toutefois laisse échapper l'occasion, et ce sera son fils Penthilos qui, ayant obtenu une réponse identique, accomplira la prophétie ${ }^{39}$. Les Thessaliens prient la pythie de choisir, parmi les fèves, le nom d'un roi. Quand la fève d'Aleuas, ajoutée à l'insu de tous par son oncle, sort du lot, les Thessaliens croient à une erreur. La pythie confirme son choix lors d'une nouvelle ambassade : «Oui! Je t'ai dit le rouquin, l'enfant qu'a porté Archédiké $»^{40}$. Une superbe inscription de Magnésie du Méandre conte l'histoire d'anciens captifs magnésiens : la pythie leur prédit le retour quand ils apercevront un corbeau blanc. Une fois ce prodige accompli, ils retournent à Delphes et demandent s'ils doivent rentrer chez eux ou s'installer ailleurs : le dieu leur propose de ne pas retourner dans leur patrie, mais de se rendre dans une Magnésie qui n'est nullement inférieure à celle du Pénée et du Pélion. Les consultants s'informent derechef : «où aller et comment?». Et Apollon de répondre qu'un homme, à la porte du temple, les guidera vers le pays des Pamphyliens, près du Méandre, où gloire et victoire les attendent. Les Magnésiens exigent encore de savoir qui est l'homme en question, et ne se mettent en route qu'après une ultime vérification. Au total, c'est à cinq reprises qu'ils auraient sollicité Delphes, en deux consultations distinctes, quatre questions de précision étant regroupées lors de la seconde visite ${ }^{41}$. Enfin les habitants de Potnia, près de Thèbes, avaient un jour tué le prêtre de Dionysos. Face au fléau qui s'ensuivit, un verdict delphique les avait condamnés au sacrifice annuel du plus beau jeune homme au dieu. Quelques années plus tard,

\footnotetext{
${ }^{37}$ Fontenrose, o.c. (n. 9), L 41 : entre autres Pausanias, IX, 38, 3.

${ }^{38}$ Fontenrose, o.c. (n. 9), L 64 : Oinomaos apud Eusèbe de Césarée, Préparation évangélique V, 20; schol. ad Théocrite, 5, 83 (= Théopompe, FrGrHist, 115 F 357).

${ }^{39}$ Fontenrose, o.c. (n. 9), L 73 : schol. ad Euripide, Rhesos, 251 (= Démon, Fr.Gr.Hist., 327 F 17); schol. ad Lycophron, Alexandra, 1374; Photios, Lexikon, Hesychios et Souda, s.v. Eschatos musôn plein.

${ }^{40}$ Fontenrose, o.c. (n. 9), L 162 : Plutarque, De l'amour fraternel, 21, p. 492ab.

${ }^{41}$ Fontenrose, o.c. (n. 9), L 163-167 : IM, 17 (aussi Hermesianax apud Parthenios, Histoires d'amour, 5, 6).
} 
«le dieu leur dit» de remplacer le garçon par une chèvre ${ }^{42}$. L'histoire, ramassée par Pausanias, illustre un cas typique : en matière de sacrifices humains révolus, les Grecs se sont toujours couverts d'une injonction oraculaire. On ignore si le second oracle fut spontané ou demandé par les gens de Potnia, et là n'était pas l'essentiel, au contraire du résultat, l'obtention d'un rituel de substitution au sacrifice humain ${ }^{43}$. Dans l'optique qui nous concerne, il s'agit de consultations solidaires, même si elles ne s'inscrivent pas nécessairement dans la logique de préciser l'oracle précédemment rendu. En conclusion, sur dix cas de consultations légendaires multiples, seuls trois sont ambigus.

Le tableau est identique pour les faits «historiques » de loin antérieurs aux sources qui les mentionnent. Trois consultations multiples seulement sont ambiguës sur un total de vingt. À tout seigneur tout honneur : la célèbre consultation athénienne sur la guerre qui débutait avec les Perses, juste avant 480 av. J.-C. Un premier oracle, atroce, semble annoncer aux Athéniens la destruction finale. Les envoyés somment alors la pythie d'en délivrer un second, guère plus engageant à première vue, avant que Thémistocle ne le décode, au nez et à la barbe des chresmologues spécialistes et des anciens qui se fourvoyaient: le «mur de bois » était la flotte, et Salamine le lieu de la victoire ${ }^{44}$. L'ambiguïté y atteint un sommet inégalable, et on ne dira jamais assez l'influence de ce récit hors pair d'Hérodote sur les mentalités anciennes et plus encore sur les esprits modernes. Il s'agit sans doute du plus bel oracle post eventum du monde grec, mais on laissera ce point de côté. Un autre cas d'ambiguïté provient du même Hérodote, mais pour l'oracle de Didymes. Les Perses ayant exigé la livraison d'un traître, Pactyès, réfugié en suppliant à Kymé, la polis des Kyméens demanda à Apollon «ce qu'ils devaient faire pour plaire aux dieux ». Réponse leur fut donnée de livrer Pactyès aux Perses. Parmi les envoyés, Aristodicos s'en étonne et reformule la question : « jusqu'à présent, bien que nous redoutions la puissance des Perses, nous avons hésité à livrer le suppliant avant d'avoir clairement appris de toi ce que nous devons faire. » Apollon confirme son verdict. Sur ces paroles, les Kyméens se préparent à obéir, mais Aristodicos, toujours insatisfait, entre dans le temple d'Apollon et en chasse tous les oiseaux. Au dieu qui, par une voix sortant de l'adyton, l'accuse d'impiété, lui qui expulse les « suppliants » du temple, Aristodicos s'étonne de l'inconséquence d'Apollon qui défend ses suppliants tout en ordonnant d'en livrer un autre. Mais le dieu s'explique ensuite : les Kyméens, rien qu'en posant une question que les nomizomena réprouvent, ont agi de façon impie, et méritent de s'attirer la colère divine par l'expulsion insensée de Pactyès ${ }^{45}$. Aristodicos apparait comme un autre

${ }^{42}$ Fontenrose, o.c. (n. 9), L 173-174 : Pausanias, IX, 8, 2.

43 Voir P. Bonnechere, Le sacrifice bumain en Grèce ancienne, Athènes/Liège, 1994 (Kernos, suppl. 3), p. 125-127.

${ }^{44}$ Fontenrose, o.c. (n. 9), Q 146-147 : en part. Hérodote, VII, 140-146.

${ }^{45}$ Fontenrose, o.c. (n. 24), nos (Q) 38-39. 
Thémistocle, un homme clairvoyant qui contraste avec l'aveugle Crésus, histoire dont nous reviendrons plus tard sur les fameuses énigmes.

Ces exemples exceptés, les autres doubles consultations dans cette catégorie de sources ne sont pas ambiguës. À la pythie qui vient d'autoriser sa réthra ${ }^{46}$, Lycurgue pose une seconde question sur le meilleur chemin pour diriger les hommes, un corrélat logique à la loi approuvée par l'oracle. Myskellos de Ripai aimerait avoir des enfants : Apollon lui en promet à condition de fonder Crotone. Myskellos s'enquiert aussitôt de Crotone, puis dans une troisième question tente de faire pencher le dieu en faveur de Sybaris, pour n'obtenir qu'une fin de nonrecevoir ${ }^{47}$. Selon une autre version, le même Myskellos se trouve à Delphes en même temps qu'Archias de Corinthe, et tous deux demandent au dieu quelle terre coloniser. Apollon répond qu'ils doivent choisir entre richesse et santé. Archias, optant pour la richesse, sera envoyé à Syracuse, tandis que Myskellos, choisissant sa santé et celle de sa cité, ira à Crotone ${ }^{48}$. Phalantos demande à Delphes s'il pourra s'installer entre Sicyone et Corinthe, mais Apollon l'envoie à Tarente. La pythie prononce un second arrêt lorsque le premier s'avère crypté à l'excès pour être compris. L'histoire légendaire des Magnésiens a sa contrepartie «quasihistorique » dans la colonisation de Cyrène par Battos de Théra. Son père, puis la cité, s'étaient vu ordonner de fonder une cité en Libye. Battos lui-même consulte l'oracle à trois reprises sur la fondation difficile de Cyrène, pour un total de sept consultations ${ }^{49}$. L'histoire est marquée par un entêtement à ne pas obéir pleinement à la volonté divine, claire depuis le début, et qu'à chaque fois Apollon réaffirme, au besoin avec humour. La consultation des Épidauriens sur l'érection des statues de Damia et Auxesia, en tous points conforme aux questions à l'authenticité prouvée, donne lieu à une précision sur le matériau à employer : pierre ou métal 50 ? Précision du même ordre dans la consultation des Spartiates en difficulté face à Tégée : quand la pythie leur révèle de retrouver les ossements d'Oreste, les Lacédémoniens s'empressent de demander où ils pourront les trouver $^{51}$. Histoire identique pour les Athéniens sommés de découvrir les ossements

46 Fontenrose, o.c. (n. 9), Q 8-9. Dans le récit de Diodore (VII, 12, 1-2), il y a même une consultation de plus : Q 7.

${ }^{47}$ Fontenrose, o.c. (n. 9), Q 28-30 : en part. Diodore, VIII, 17, 1-2.

${ }^{48}$ Fontenrose, o.c. (n. 9), Q 31 : en part. Strabon, VI, 2, 4 et Élien, fr. 316.

49 Fontenrose, o.c. (n. 9), Q 45-51 (et p. 120-123) : en part. Hérodote, IV, 150-159. Sept consultations sans compter les demandes d'explications non exaucées par le dieu. Voir C. Calame, Mythe et bistoire dans l'antiquité grecque. La création symbolique d'une colonie, Paris, $2011^{2}$.

${ }^{50}$ Fontenrose, o.c. (n. 9), Q 63 : Hérodote, V, 81, 1-2. La remarque de Fontenrose (p. 289), à l'effet que la double consultation «arouses suspicions », est par contre totalement erronée puisque l'usage est bien attesté. La réponse du dieu, qui opte pour une troisième possibilité, le bois, que les hommes dans leur sagesse limitée n'avaient pas entrevue, laisse planer un doute important sur l'historicité de l'épisode.

${ }^{51}$ Fontenrose, o.c. (n. 9), Q 89-90 : Hérodote, I, 67; Diodore, IX, 36, 3; Pausanias, III, 3, 6. 
de Thésée ${ }^{52}$, ou encore pour les Déliens qui, expulsés de Délos, se voient intimer de trouver le lieu de naissance d'Apollon : surpris d'apprendre que ce lieu n'était pas Délos, ils interrogent le dieu encore, qui leur annonce qu'un corbeau les guidera ${ }^{53}$. Les Thraces Dolonces, en difficulté dans la guerre qui les opposait aux Thraces Apsinthiens (ou l'inverse), doivent, leur dit la pythie, prendre pour œeciste le premier homme qui leur offrira l'hospitalité. Traversant l'Attique, ils sont invités par Miltiade, fils de Cypsélos, et lui révèlent la prophétie. Ce dernier vérifie aussitôt les dires des Thraces à Delphes, qui l'engage à les suivre ${ }^{54}$. S'enquérant du sort que la vie réservera à ses fils, Deinoménès apprend que tous trois seront tyrans. Ce à quoi il ajoute : "sans doute pour leur malheur, seigneur Apollon », et le dieu confirme le trait d'esprit, comme une klèdôn ${ }^{55}$. Pour avoir jeté à la mer la statue de Théagène, les Thasiens souffrent d'un fléau. La pythie les invite à rappeler tous les bannis, mais - la mesure restant inefficace - elle révèle qu'il leur faut aussi récupérer la statue, qu'un pêcheur ramènera miraculeusement ${ }^{56}$. D’après Plutarque, la défaite de Chéronée avait été annoncée par plusieurs oracles de la pythie, ce qui implique une répétition, le tout accompagné d'autres signes et d'un oracle sibyllin ${ }^{57}$. Bien plus tard, l'Histoire anguste se fait le relais d'une triple interrogation à propos du successeur de Commode, de toute évidence calquée sur la réponse pythique à la question du plus sage des hommes, Socrate : suite à la réponse «Fuscus (Sévère) est le meilleur, (Pescennius) Niger est bon, (Clodius) Alb(in)us est mauvais ", la pythie prédit la mort sanglante des deux derniers et l'avènement d'un empereur africain. Celui-ci, lors d'une troisième question, est promis à un long règne ${ }^{58}$. Dans l'antiquité chrétienne enfin, on s'imagine Auguste questionner Apollon sur son successeur, rapidement forcé de reformuler sa demande: "pourquoi l'oracle demeure-t-il silencieux?». Et Apollon, soudain loquace, d'expliquer son silence depuis que le Christ l'a prié de quitter son temple et de retourner dans l'Hadès ${ }^{59}$.

\footnotetext{
${ }^{52}$ Fontenrose, o.c. (n. 9), Q 164 : en part. Plutarque, Thésée, 36, 1; Cimon, 8, 6; Pausanias, III, 3, 7. Voir le récit analogue sur les ossements d'Hésiode, L 42 (et supra, n. 37).

${ }^{53}$ Fontenrose, o.c. (n. 9), Q 191: Plutarque, Les oracles de la pytbie, 5, p. 412c. Le second oracle est un double entendre, car c'est une hôtelière nommée "Koroné », Corneille, qui leur livre inconsciemment la clef de l'énigme : le dieu est né à Tégyre.

${ }^{54}$ Fontenrose, o.c. (n. 9), Q 109-110 : en part. Hérodote, VI, 34-36.

55 Fontenrose, o.c. (n. 9), Q 140 : Plutarque, Les oracles de la Pythie, 19, p. 403c.

${ }^{56}$ Fontenrose, o.c. (n. 9), Q 170-171 : surtout Dion Chrysostome, 31, 97 et Pausanias, VI, 11, 7-8.

57 Fontenrose, o.c. (n. 9), H 22 : Démosthène, 19, 1 (la classification de H 22 dans les cas « historiques » est abusive : il devrait s'agir d'un Q).

${ }^{58}$ Fontenrose, o.c. (n. 9), Q 253-256 : Aelianus Spartanus, Histoire auguste, Pescennius Niger, 8 (avec jeu oraculaire sur les couleurs). Cf. «Quelqu'un est-il plus sage que Socrate? » et une des réponses attribuées à la pythie : "Sophocle est sage, Euripide plus encore, mais de tous les hommes, c'est Socrate qui est le plus sage » (sources chez FONTENROSE, o.c., H 3).

${ }^{59}$ Fontenrose, o.c. (n. 9), Q 250 : en part. Eusèbe de Césarée apud Cedrenos, 1, p. 320b; Souda, s.v. Angoustos.
} 
Il valait la peine de détailler tous les cas imaginaires pour se faire une idée exacte du phénomène dans les mentalités, avant d'aborder les cas dont on est assuré de l'historicité. Au total donc, sur trente-deux cas issus de l'imaginaire grec des oracles, seuls six sont dus à l'ambiguïté, trois pour les questions "quasi-historiques », trois pour les cas légendaires. Et encore les ambiguïtés vicieuses y sont-elles rarissimes : si le cas le plus net est celui du « mur de bois », il est aussi le plus dramatiquement isolé, et on comprend difficilement pourquoi il est si souvent érigé en exemple.

\section{Dans les réponses contemporaines aux faits, littéraires ou épigraphiques}

Les réponses contemporaines des faits ne sont pas en reste, épigraphie et littérature confondues. Leur distribution chronologique est remarquable, du $\mathrm{VI}^{\mathrm{e}}$ siècle av. J.-C. à l'époque romaine, ce qui suppose une tradition bien enracinée : trois à Delphes, cinq à Dodone, six à Didymes, et plusieurs cas chez Trophonios, sans oublier Asclépios et Sarapis. Je passe en revue, par ordre chronologique, les cas privés et publics les plus clairs ${ }^{60}$. La documentation est certes disparate, mais n'est-ce pas là le signe de ce que la pratique était répandue dans tous les champs de l'activité oraculaire?

\subsection{Cas privés}

2.1.1. La première inscription connue de l'oracle de Didymes, vers 600-550 av. J.-C., et aussi l'une des plus anciennes réponses oraculaires attestées, sinon la première, semble ainsi être un complément à une consultation antérieure.

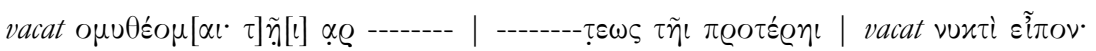

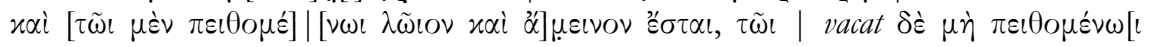

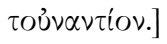

(...) ce que j'ai dit la nuit passée. Il est meilleur et plus avantageux pour lui d'obéir, et le contraire s'il n'obéit pas.

$60 \mathrm{Il}$ existe des cas incertains. On note, dans le domaine privé, une inscription de Didymes, datée du II $\mathrm{e}^{\mathrm{e}}$ ap. J.-C. (FonTENRosE, o.c. [n. 24], A 4), par trop fragmentaire : « [Les Ph]ilodionysoi --| ... à un consultant qui [---] tout récemment [--- au di]eu ». La réponse est presque perdue :

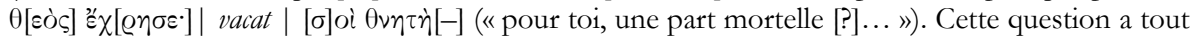
l'air, étant donné les attendus spécifiés au dieu, d'une précision sur le culte dionysiaque. Fontenrose, o.c. (n. 24), (H) 20, est une consultation par un certain Appheion au sujet d'un numéro de tauromachie. Il demande protection au dieu, et précise qu'Apollon et ses dieux ancestraux l'ont toujours épaulé jusque-là : il est possible qu'il ait consulté plusieurs fois, voire chaque fois qu'il se produisait aux jeux dans la région, mais on pourrait y voir aussi une simple dévotion. En fait, de toute façon, il s'agit plus de consultations systématiques sur la réussite de chaque numéro que de consultations multiples sur un seul et même fait. 
La perte du début de cette inscription (boustrophédon) nous prive de tout contexte. L'oracle est en outre fragmentaire mais les restitutions sont raisonna-

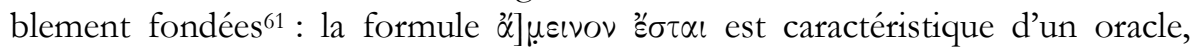
même à jamais perdu.

2.1.2. Vers 450-425 av. J.-C., à Dodone, un certain « Arizèlos redemande au dieu ce qu'il doit faire et accomplir qui lui soit meilleur et plus avantageux, et comment il pourra acquérir beaucoup d'argent » (A@i

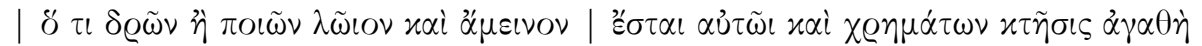
$\varepsilon ̋ \sigma \tau l)^{62}$. Soit une première réponse n'a pas été satisfaisante à ses yeux, et lors de la même visite, il tente à nouveau sa chance; soit il revient à Dodone après avoir tenté de devenir riche, sans que la première réponse de Zeus ait fait de lui un second Crésus. On ne sait laquelle des deux options choisir, avec leurs avenues différentes, mais la seconde possibilité est plus réaliste. Dans le cas d'une précision immédiate demandée au dieu, pareil comportement manifesterait une touche d'audace, mais sans excès. Dans le cas d'un échec passé, la nouvelle demande manifesterait la foi malgré tout conservée dans l'oracle, et la révérence (au moins en surface) du pèlerin qui se garde bien de reproches directs. Quoi qu'il en soit, Arizèlos demande une précision sur un oracle déjà rendu sans que nulle ambiguité ne motive sa démarche, ambiguité qui au demeurant n'apparait nulle part dans les questions dodonéennes.

2.1.3. En 401 av. J.-C., Socrate aurait reproché à Xénophon d'avoir triché, en demandant au dieu de Delphes comment revenir en santé et glorieux de l'expédition perse contre Artaxerxès. Il eût dû interroger Apollon d'abord sur l'opportunité de s'en aller ou de rester ${ }^{63}$. Ce qui aurait impliqué une seconde question, posée dès réception de la première. Ce passage socratique est très intéressant, en ce qu'il est toujours accepté comme authentique par la critique moderne. On dénie à l'auteur, en quelque sorte, toute part d'élaboration littéraire, alors que nous sommes dans un "dialogue socratique ${ }^{64}$, et qu'il en émane justement une remarquable force de persuasion qui a échappé à tous les lecteurs. Nous reviendrons à Xénophon et ce type de consultation dans la partie consacrée aux consultations publiques.

${ }^{61}$ Fontenrose, o.c. (n. 24), (H) 1. A. Rehm (Milet I, 3, 178) reconstitue, tout au début:

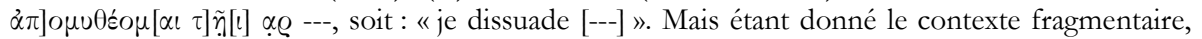
c'est peut-être un peu trop conjectural.

62 Pour Dodone, je suis systématiquement l'édition et la traduction (parfois légèrement modifiée) de LHÔTE, o.c. (n. 1), ici no 107A. Selon lui, Arizèlos s'exprime avec une certaine recherche qui correspond à sa belle écriture. Le terme ė $\pi \alpha v \varepsilon \varrho \omega \tau \tilde{\alpha} \omega$ signifie soit «interroger de nouveau », soit "demander ensuite»: voir LSJ ad loc. et suppl. (par exemple Démétrios de Phalère, Du style, 288). On pourrait donc aussi traduire : « Arizèlos 'demande ensuite' au dieu (...). En ce cas, la première question aurait pu concerner un autre sujet.

${ }^{63}$ Fontenrose, o.c. (n. 9), H 11 : Xénophon, Anabase III, 1, 4-8.

${ }^{64}$ Voir infra, p. $90-91$. 
2.1.4. Vers 400 av. J.-C., à Dodone, un consultant rédige une question passablement alambiquée, dont Éric Lhôte a courageusement tenté une traduction: «Au sujet de la répo]nse (de l'oracle), et des sceaux des 'puis[oirs', sceaux qu'Ais[chillos] n'a pas apposés ni refermés, [est-ce que, s'étant rendu] à $<$ Do $>$ done au sujet de la lamelle, [Untel] a poussé [Aischi]llos à ne pas même fabri[quer les sceaux, à ne pas] les graver, à ne pas les apposer? ». Déjà obscur, le

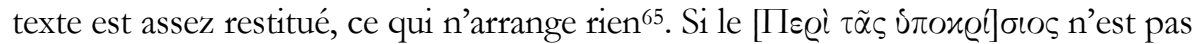

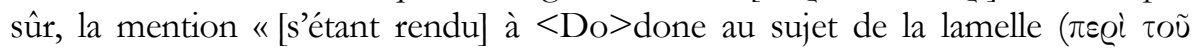

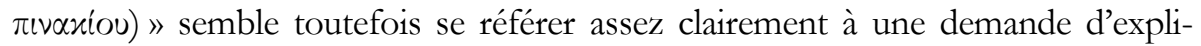
cation.

2.1.5. Sur une des rares lamelles de Dodone à comporter, au verso, ce qui semble la réponse à la question posée - les deux faces sont de la même main - et datée de 400-390 av. J.-C.66, on lit: "Engager son capital dans le grand commerce, sur terre et sur mer, pendant autant de temps que Timodamos en décidera lui-même, sont-ce là les actions les plus fortes? $\aleph^{67}$. Le dieu semble avoir répondu : «Habiter en ville, $\mathrm{y}$ faire du petit et du grand commerce, mais céder tes parts de cargo. Faire du grand commerce en important, et en exportant des marchandises sur terre et sur mer, en vendant et en achetant ». La réponse a l'air trop complexe pour avoir été délivrée en une fois, mais comme le texte est unique, et non exempt d'imprécisions, il vaut mieux laisser le cas en suspens.

2.1.6. Désireux d'établir un culte d'Archiloque, un certain Mnésiépès de Paros a consulté trois fois l'oracle de Delphes. Tout un dossier a été gravé sur pierre vers 250-225 av. J.-C., avec une vie d'Archiloque et deux oracles «anciens ", en vers, suivis des trois réponses faites à Mnésiépès (dont les questions sont absentes $)^{68}:$ (1) «Il est meilleur et plus avantageux d'élever un autel dans le temenos qu'il est en train de construire, et d'y accomplir un sacrifice aux Muses, à Apollon Musagète, Mnémosyne, ainsi qu'à Zeus Hyperdexios, Athéna Hyperdexia, Poséidon Asphaleios, Héraclès, Artémis Eucleia, et d'envoyer une action de grâce à l'Apollon de Pythô ». (2) «Il est meilleur d'élever un autel dans le temenos et d'y sacrifier à Dionysos, aux Nymphes, aux Heures, ainsi qu’à Apollon Prostatérios, Poséidon Asphaleios, Héraclès, et d'envoyer une action

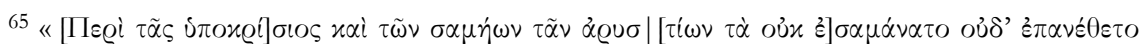

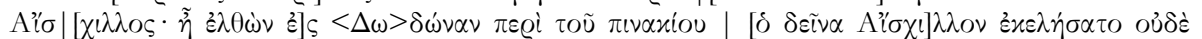

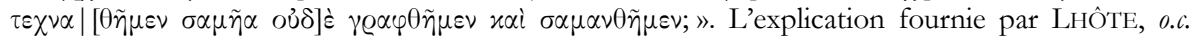
(n. 1), ad 134 (en relation avec Cicéron, Sur la divination I, 34, 76 et II, 32, 69), n'est pas convaincante, même si je n'en ai pas d'autre à proposer.

${ }^{66}$ LHÔTE, o.c. (n. 1), no $95 \mathrm{AB}$.

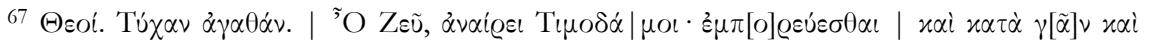

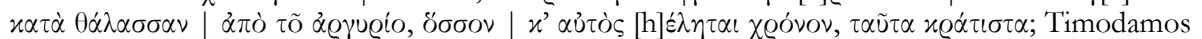
est, à ma connaissance, le seul consultant qui ose exiger une précision presque hybristique de la réponse, en s'adressant au superlatif plutôt qu'au traditionnel comparatif.

68 Fontenrose, o.c. (n. 9), H 74. Voir Amandry, o.c. (n. 9), p. 206-207 et n. 35. SEG 52 (2002), 872. 
de grâce à l'Apollon de Pythô ». (3) «Il est meilleur d'honorer le poète Archiloque ainsi que le propose Mnésiépès ». Ces trois réponses concernent un même culte et résultent de questions très semblables, même si l'on ne peut être sûr qu'elles furent posées d'affilée et même s'il subsiste un doute quant à savoir s'il s'agit vraiment de questions d'approfondissement.

2.1.7. Les consultations successives sont courantes dans les sanctuaires médicaux : à l'époque hellénistique tardive, à Pergame, le pèlerin d'Asclépios demandait au besoin des précisions sur un rêve précédent en s'acquittant d'un droit réduit ${ }^{69}$, un porcelet à la place de deux ${ }^{70}$. Les oracles à répétition constituent évidemment un des ressorts des consultations médicales par les hypocondriaques, tel Aélius Aristide, dont les discours sacrés ne sont qu'une suite de prescriptions oraculaires et médicales, un échange presque ininterrompu entre le dieu et l'homme malade ou persuadé de l'être. Il existe certaines relations épigraphiques des mêmes phénomènes : par exemple à Épidaure, la stèle de Marcus Iulius Apella de Mylasa, vers 160 ap. J.-C. ${ }^{71}$. Le malade décrit les neuf jours passés au sanctuaire à recevoir des injonctions, avant d'être soigné par le dieu en personne dans une vision, voire plusieurs. Prétendre que les pèlerins pouvaient tricher en alléguant aux prêtres, des mois ou des années plus tard, que leur nouvelle demande était la suite d'un oracle rendu par le passé, n'est guère satisfaisant: abuser les prêtres c'est une chose, abuser Asclépios quand on lui demande la guérison par contact onirique en est une autre ${ }^{72}$. Les inscriptions de miracles à Épidaure renferment aussi leur lot de rêves successifs ${ }^{73}$.

2.1.8. Dodone, entre 300 et 167 av. J.-C. ${ }^{74}$, livre un autre exemple, laconique

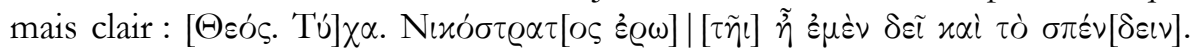
"Nikostratos demande à l'oracle s'il faut aussi lui en faire une libation ». Le $x \alpha i$ implique une première réponse que le consultant désire préciser. Ce genre de

69 Voir l'édition et la datation de M. WörRLE, «Die Lex sacra von der Hallenstrasse (inv. 1965, 20) », in C. НАВICHT, Die Inschriften von Pergamon, Berlin, 1969 (Altertümer von Pergamon, 8, 3), p. 167-190.

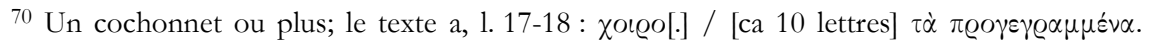

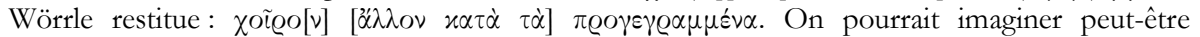
$\chi o i \varrho o[u c]$ et un chiffre (je n’ai pas vu la photographie de la pierre). Notons la même incertitude que pour Arizèlos à Dodone quant au moment du second oracle : lors de la même occasion, ou après un long intervalle? no 432).

71 IG IV2, 126 (= J. et L. EdELSTEIN, Asclepius, Baltimore/Londres, 1945 [réimpr. 1998],

72 Les menteurs compulsifs existent à toutes les époques, mais il s'agit là de cas psychopathologiques, qu'il ne faut pas généraliser à toute la population.

73 Je les place ici, dans les consultations « réelles » et non imaginaires, par simple facilité. Dans l'édition de L. LiDonnici, The Epidaurian Miracle Inscriptions, Atlanta, 1995 : A2, A17, B2, B3, B5. On note aussi que bien des gens dormaient plusieurs nuits de suite dans l'attente du rêve salvateur. Chaque nuit passée dans l'abaton peut en fait correspondre à une même question posée.

${ }^{74}$ LHÔTE, o.c. (n. 1), no $10 \mathrm{Ab}$. 
précision sur un acte rituel prescrit semble devenir plus fréquent à l'époque hellénistique et romaine, surtout dans la sphère publique en Asie Mineure.

2.1.9. Ainsi, dans un autre lieu, à une autre époque, les mêmes procédures apparaissent de rigueur. À Didymes, une consultante s'adresse à Apollon, à la fin du II ${ }^{\mathrm{e}}$ siècle ap. J.-C. : «[puisque, en ton divin oracle,] tu lui as répondu

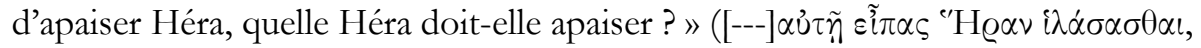
$\left.\pi o^{\prime} \alpha \nu \mid i \lambda \alpha \sigma \eta \tau \alpha l ;\right)^{75}$. La première question est perdue, mais la seconde interrogation en a conservé, sinon la réponse, au moins le contenu. Et le dieu de préciser, dans l'amphigouri versifié typique de l'époque tardive, le lieu de culte de l'Héra en question ${ }^{76}$.

2.1.10. Un peu plus tard, au III e siècle ap. J.-C., le prophète Ulpianos, dévot, consulte lui-même l'oracle. L'inscription, qui parle d'autels et de tables sacrificielles, est certes mutilée ${ }^{77}$, mais la séquence est nette : suite à la réponse de l'oracle, le prophète demande au dieu de spécifier " où placer la [table] sacrée » pour l'honorer comme il sied. Il est possible que les deux interventions oraculaires aient été motivées par une vision, ou un rêve, que le prophète aurait voulu vérifier par le biais du premier oracle, mais le texte est trop allusif pour en être sûr $^{78}$.

2.1.11. À la fin du III e siècle ap. J.-C., enfin, un autre prophète, Damianos, s'enquiert d'une précision: "puisque par ton saint oracle tu l'as autorisé à installer, dans ton cercle d'autels consacrés, un autel de sa déesse ancestrale la plus sainte, Korè Sôteira, près de l'autel de Déméter la plus auguste, Karpophoros, d'agir en nomothète quant à l'épiclèse hymnique et de bon augure. » Et Apollon de choisir « Sôteira Meilichos ${ }^{79}$.

${ }^{75}$ Fontenrose, o.c. (n. 24), (H) 24. L. ROBERT (CRAI, 1968, p. 578, n. 1) préférait la lecture

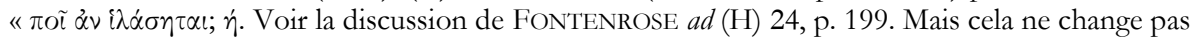
grand-chose pour notre propos.

76 «Là où le grand âge, vénérable de par ses longues années, a l'usage de baigner ses corps honorables de vieillesse, là où les jeunes filles, encore non initiées aux chambres nuptiales, forment leur chœur au beau rythme de la mélodie harmonieuse de la flûte, dans les palais d'un

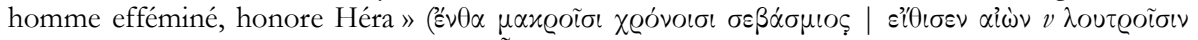

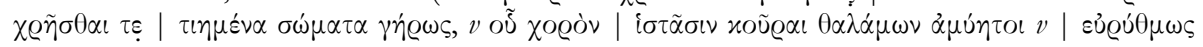

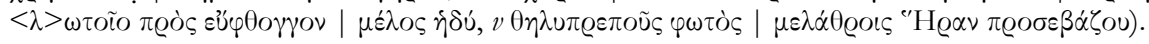

77 Fontenrose, o.c. (n. 24), (H) 29 : «Comme un arbre d'envergure, tu as des branches robustes, et tu pais des moutons à la laine épaisse. Il est juste dès lors que je réponde à nouveau à ta question. Oui, tu as obéi à mes oracles, en augmentant ta sage piété à croire les dieux lors d'interrogations et de réponses précédentes, tu as dirigé ton esprit vers les autels pour les

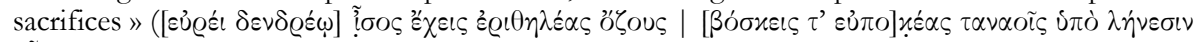

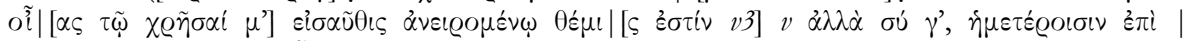

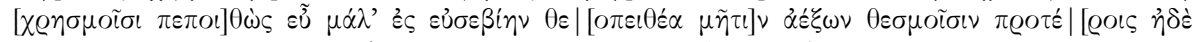

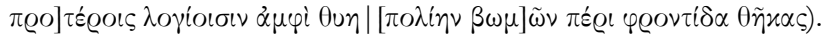

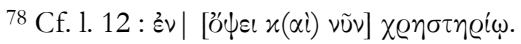

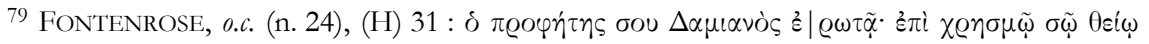

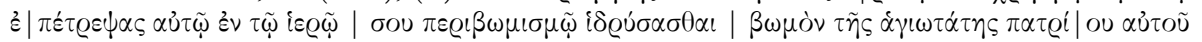

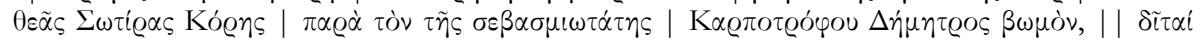


2.1.12. [annexe] En 258/257 av. J.-C., Zoïlos d'Aspendos, recommandé par les philoi royaux, travaille pour Apollonios, le fameux dioicète de Ptolémée II. Il interroge par incubation Sarapis sur la santé de son supérieur et son succès auprès de Ptolémée. À plusieurs reprises, le dieu lui fait savoir qu'Apollonios devrait lui bâtir un enclos dans le quartier grec près du port (de Memphis), avec un prêtre spécialement appointé. Zoillos, qui tente d'échapper à la prescription, est puni par le dieu d'une maladie, pour laquelle il doit à nouveau incuber et recevoir le même ordre. Au moment de sa guérison, un quidam cnidien entreprend la construction du sanctuaire, mais Sarapis lui interdit en rêve de poursuivre, de sorte que le temple soit édifié expressément par le dioicète. La dépense ne doit pas être un obstacle, conclut Zoillos, puisque l'action revaudra au bienfaiteur toute l'attention du dieu, tant en ce qui concerne sa santé que dans ses relations avec le monarque ${ }^{80}$. J'ai placé le cas en annexe, car il est un peu différent des autres : Zoillos, qui consulte pour un motif général, se fait dire à plusieurs reprises d'accomplir une action pour laquelle il n'a pas posé de question $^{81}$. Les rêves à répétition ne sont pas sans rappeler les oracles de la tradition littéraire, où la pythie répète inlassablement la même réponse quelle que soit la question posée ${ }^{82}$. Si la lettre est authentique, et si le sentiment religieux qu'on y trouve l'est tout autant, cela n'élimine pas les influences littéraires sur les mentalités, ou dans un argumentaire conçu comme acceptable.

\subsection{Cas publics}

Du côté des pouvoirs politiques, les exemples sont plus rares, mais comme en moyenne les grands oracles, dès le moment où apparaissent les sources contemporaines des faits, se consacrent à 90\% aux enquêtes privées, ne laissant aux consultations officielles que 10 maigres pour cent, la surprise n'en est pas

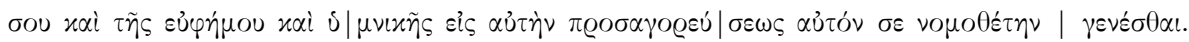

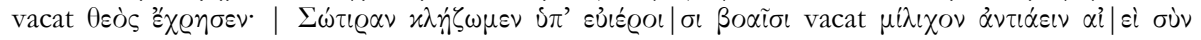

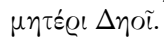

80 PSI IV, 435 (= P. Cair. Zén, 50034). W. Clarysse, K. Vandorpe, Zénon. Un homme d'affaires, Louvain, 1995, p. 78-85. Le point par K.J. RigSBY, "Founding a Sarapeum », GRBS 42 (2001), p. 117-124. Le texte y est relu selon une optique minimaliste qui est très séduisante. Zoillos tente, sur l'ordre du dieu, de faire construire un petit sanctuaire, sans qu'il faille y voir d'autre but grandiose, social ou politique. Dans mon article sur les consultations multiples dans des sanctuaires différents, j'avais cru qu'on pouvait déceler plusieurs Sarapeia consultés par l'employé, mais il n'en est rien (l.c. [n. 30], p. 126).

81 P.Oxy. XI, 1381 (Arétalogie d'Imouthès-Asclépios) est un autre exemple bien connu d'un dieu qui force le consultant à obéir, par la maladie et les apparitions : là, un auteur qui s'est vu intimer la traduction grecque d'un « original » égyptien, avait de même traîné dans l'accomplissement de l'ordre divin. Ces manquements successifs sont déjà typiques de la chronique de Lindos (voir supra n. 28), datée du Ier s. av. J.-C.

${ }^{82}$ Par exemple Fontenrose, o.c. (n. 9), Q 124 (Sparte et la chute des Pisistratides). 
vraiment une ${ }^{83}$. Deux cas sont hors de doute, et deux autres hautement probables. Tous corroborent les informations sur les consultations doubles obtenues dans des sanctuaires différents ${ }^{84}$.

2.2.1. Au IVe siècle av. J.-C., à Dodone, de futurs (?) colons demandent : « À propos de leur installation au pays des Chemarioi, doivent-ils s'installer 'à cet endroit même' $»^{85}$, ce qui sous-entend une seconde question sur un oracle antérieur. Il n'est pas nécessaire de voir dans le manteion un moteur de la colonisation qui assigne des places nouvelles à civiliser. Bien plutôt, les colons ont consulté l'oracle, par tradition, en demandant s'il était favorable de s'installer à tel endroit qu'ils avaient sélectionné, en l'occurrence le pays des Chemarioi. L'oracle a dit oui. Un problème se pose, et les colons de revenir en demandant s'il est vraiment nécessaire de s'installer à cet endroit précis ${ }^{86}$.

2.2.2. Xénophon, à la fin des Poroi, vers 355 av. J.-C., suggère aux Athéniens de soumettre ses propositions de réformes financières, après un vote favorable à l'ecclesia, à deux sanctuaires oraculaires, Delphes et Dodone. Ensuite, si Apollon et Zeus se prononcent en faveur des idées déjà entérinées par le vote athénien, on leur demanderait à quels dieux sacrifier pour que l'entreprise connaisse le meilleur succès. Que cette proposition à l'ecclésia ait été suivie d'effet ou non, elle implique donc deux doubles consultations parallèles sur le fameux modèle «socratique » discuté plus haut. D’aucuns concluront que Xénophon, un peu obtus dans sa jeunesse, avait fini par assimiler la morale de son maître. J'y verrais bien davantage la preuve de ce que Xénophon, persuadé que la piété voulût qu'on ne forçât en rien les dieux, avait authentifié son raisonnement au sceau d'une maxime socratique dans l'Anabase: en confessant une «erreur de jeunesse » qui focalisait la critique des lecteurs sur lui, il mettait en place un redoutable piège rhétorique pour forcer ces mêmes lecteurs ou auditeurs à

83 Ces statistiques proviennent de Dodone, en tenant compte du ratio privé/public des lamelles de plomb. Celles-ci, de par leur côté bon marché, donnent une bien meilleure idée de la réalité que les autres oracles comme Delphes ou Didymes, ou Claros, dont on a conservé essentiellement les inscriptions sur pierre, et donc coûteuses et d'intérêt général, public. Je reviendrai ailleurs sur ces questions (en attendant, je renvoie à mon article cité n. 2).

${ }^{84}$ Voir supra, n. 30. D'autres cas sont moins probants. FonTENROSE, o.c. (n. 9), H 44 est un hybride : en fait, l'inscription indique quatre désignations de prêtres (Aphrodite, 1. 5, 74 et 160 ; Déméter et Coré, 1. 89; Dionysos, 1. 145) par voie oraculaire (cf. le terme pythochrestos qui suit chaque nom de dieu). Fontenrose considère deux possibilités : soit une consultation « en bloc ", soit quatre consultations isolées. Quoi qu'il en soit, même en bloc (et cette solution n'a pas ma préférence), la question posée n'est pas la même : il s'agit de quatre prêtrises différentes. Peut-être Fontenrose, o.c. (n. 24), (H) 23 est-il un prolongement de (H) 22 (II ap. J.-C.), mais rien ne permet d'en être sûr : il s'agit de deux oracles rendus à la même personne, Alexandra, la prêtresse de Déméter Carpophoros. Mais le nom de ladite prêtresse ne figure pas dans (H) 23, fragmentaire, et il pourrait s'agir tout autant de deux consultations indépendantes.

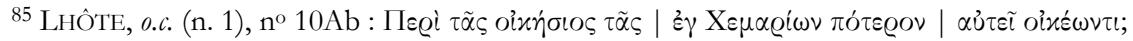

${ }^{86}$ LHÔTE, o.c. (n. 1), p. 274-275, a répertorié les toponymes qui pourraient résulter de la colonisation, suite à ces consultations. 
revoir les usages contemporains de la consultation oraculaire dans le sens d'une piété plus accomplie.

2.2.3. La plus célèbre des inscriptions de l'oracle de Trophonios à Lébadée ${ }^{87}$, datée de 350 av. J.-C., liste le nom des donateurs qui ne se seront pas contentés de verser les dix drachmes (1. 4 : " dix gâteaux de dix drachmes ») prévues pour la consultation. Dix drachmes, pour consulter un oracle, c'est un prix en soi prohibitif, et il est clair qu'on n'allait pas interroger Trophonios pour des futilités quand on n'était pas riche. Il faut ajouter à cette lourde dépense le prix des victimes, dont Pausanias, bien plus tard, dressera une liste impressionnante ${ }^{88}$. Au bas mot, la consultation devait coûter, sans inclure les frais de voyage ni le séjour d'au moins une semaine, entre 40 et 100 drachmes, en fonction des victimes sacrifiées (poulets / cochonnets ou ovins / caprins, sans même considérer la possibilité de bovins). Dans ce contexte, l'inscription prend tout son sens : les pèlerins qui paient très cher, et à intervalle très rapproché, ont peu de chance d'être des particuliers agissant pour eux-mêmes. Ils semblent plutôt mandatés par des corps constitués, cités ou koina, aux moyens et aux besoins plus conséquents, ainsi qu'aux volontés plus ostentatoires. De la sorte, le nom de Pythonicos de Tanagra revient trois fois en suivant. Il est possible que d'autres pèlerins aient consulté l'oracle entre ses trois visites, mais aucun d'eux n'avait déboursé plus des 10 drachmes requises. Pythonicos, de plus, paie 40 drachmes, 40 encore, puis 120. Évanthidas de Locride paie [20] drachmes, puis deux fois 70 , et enfin 120 . Ces consultants viennent peut-être, à chaque fois, pour des raisons différentes, mais l'idée d'une consultation à répétition, avec à la clef d'ailleurs une majoration progressive des dons en argent, reste la plus simple et la plus attirante : il s'agirait en quelque sorte d'un dialogue entre le dieu et la cité ou la ligue qui l'interroge. Si tel est le cas, il s'agit aussi de consultations politiques au sens large, civiques, aux alentours de 350 av. J.-C.

2.2.4. Cette lecture me semble trouver confirmation dans une autre inscription de Béotie, au Ptoion cette fois, vers 230-220 av. J.-C. Quatre réponses de Trophonios, rendues au Locrien Calliclidas d'Oponte ${ }^{89}$, mandaté par le koinon

${ }^{87}$ IG VII, 3055. Excellente édition critique (sur base de deux copies de voyageurs, car l'original a disparu) de F. SALVIAT et C. VATIN, « Le règlement de Lébadée sur la consultation de l'oracle de Trophonios ", in EIDEM, Inscriptions de Grèce centrale, Paris, 1971, p. 81-94. Légères retouches par A. SCHACHTER, Cults of Boiotia III, Londres, 1994, p. 87-89.

${ }^{88} \mathrm{Si}$ la continuité du rituel est attestée sur le long terme, on pourrait évidemment mettre en doute que le paiement de 10 drachmes ait persisté jusqu'à Pausanias. Quoi qu'il en soit, l'importance des sacrifices est déjà attestée par les fragments de la comédie attique, et, avec ou sans les dix drachmes, la consultation demeurait hors de prix (le détail dans mon livre, cit. n. 17, p. 57-60).

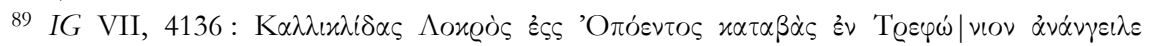

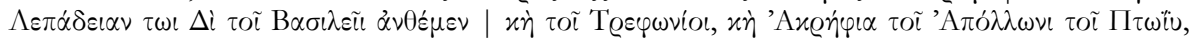

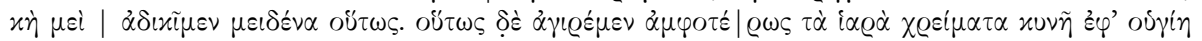

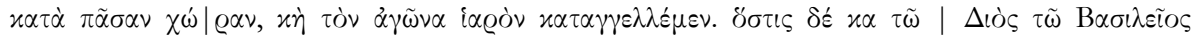

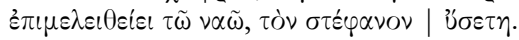


béotien, concernent successivement les points très différents d'un même dos$\operatorname{sier}^{90}$. Le dieu a déclaré (1) qu’il fallait consacrer Lébadée à Zeus Basileus et à Trophonios, et Acraiphia à Apollon Ptoios, et que personne ne devrait leur faire de tort. (2) Que les deux cités, de concert, devaient rassembler les sommes sacrées, pour le bien commun, dans tout le pays, et (3) annoncer que l'agôn était sacré. (4) Celui qui aurait eu la charge du temple de Zeus Basileus remporterait une couronne. L'idée d'un perfectionnement de la réponse, par consultations successives, semble au minimum très attractive: l'émissaire faisait rapport au koinon, qui pouvait décider d'une nouvelle question. Le mode de consultation de Trophonios, par déséquilibre physio-psychologique suite à une longue et pénible préparation vécue comme une purification, ne semble pas avoir permis la réponse à quatre questions aussi différentes en un seul face-à-face avec le dieu. Ont-elles été posées séparément dans une visite unique, ou lors de quatre voyages séparés ? Il est impossible de le dire. Mais toutes concourent à la préparation de l'asylie du Ptoion, voire du Trophonion (asylie dont nous n'avons pas d'autre trace tangible pour le moment, mais le sanctuaire de Lébadée est toujours non localisé). Certes, on pourrait faire valoir que le consultant, Calliclidas d'Oponte, était venu chercher une approbation globale au projet, mais l'inscription IG VII, 3055, avec ses consultations répétitives, oblige au minimum à laisser le dossier ouvert, surtout quand on considère que cet usage était admis dans les mentalités.

\section{Conclusions}

La double consultation dans un même sanctuaire est donc une tradition qu'on connaît de l'archaïsme à l'époque romaine, dans tous les grands oracles, publique et privée, et sur n'importe quel type de question. Les consultations légendaires et quasi-historiques confirment largement, sur le principe, la série bien documentée des documents d'époque, comme d'ailleurs les questions simultanément posées à deux manteia différents. L'examen des documents évacue toute possibilité d'évolution rationaliste ou sophistique face aux oracles qu'on aurait peu à peu remis en cause, évacue également toute cassure potentielle entre piété populaire et piété publique et aussi toute limitation à un domaine mantique particulier. Aucune de ces questions ne s'avère secrète ou dangereuse, et aucune n'aurait permis aux prophètes, prophétesses, prêtres ou prêtresses d'abuser du «client» ou de s'immiscer dans les affaires de la cité.

\footnotetext{
${ }^{90}$ Oponte, à cette date, semble ne plus faire partie du koinon, comme je l'ai écrit ailleurs, mais demeure proche de la Béotie et Calliclidas pourrait avoir été choisi pour la «neutralité » de sa cité : voir D. KNOEPFler in Bulletin épigraphique, 2007, no 311. Voir aussi P. RoesCH, Études béotiennes, Paris, 1982, p. 232-235; A. SCHACHTER, "A Consultation of Trophonios (IG VIII, 4136) ", AJPh 105 (1984), p. 258-270; K.J. RigsBy, Asylia. Territorial Inviolability in the Hellenistic World, Berkeley et al., 1996, nos 2-3 (qui penche pour un oracle rendu après la déclaration d'asylie pour le temple du Ptoion et les Ptoia, et qui viserait de nouveaux honneurs pour Apollon); P. SÁnchez, L'amphictionie des Pyles et de Delphes, Stuttgart, 2001, p. 348-349.
} 
Aucune ambiguiité enfin n'est visible dans les réponses qui appellent les questions d'approfondissement rapportées dans les sources contemporaines des faits (et même aussi dans la grande majorité des autres). D’ailleurs, à rebours, si les réponses avaient été systématiquement ambiguës, on trouverait bien davantage de questions d'éclaircissement... De tout ceci, il ressort que la double consultation, pour le consultant privé ou public, consiste seulement à préciser la réponse de l'oracle qu'il estime trop vague. $\mathrm{Ni}$ défiance, ni roublardise, ni sophistique ne me semblent à l'ordre du jour. Pourquoi ne pas voir simplement une façon pieuse d'approcher la divinité qui révèle les choses cachées ? En dépit de ce constat, lorsqu'on parle de consultations répétées, les premières qui viennent à l'esprit sont celles de Crésus et des Athéniens avant la bataille de Salamine, qui justement sont tout sauf représentatives.

Revenons un moment à l'histoire de Crésus qui ruine son empire en voulant détruire celui de Cyrus. Il y a souvent eu flottement sur la nature du double entendre, qui y est de loin moins obscur que dans l'épisode du «mur de bois», surtout quand on considère la geste de Crésus au complet, avec son étonnante série de prophéties mal comprises. En fait, si Crésus réinterroge l'oracle, après son fameux test oraculaire, et à quatre reprises, il ne le fait pas selon les voies de la sagesse : plutôt que de demander une précision, il saute aux conclusions et interroge Apollon sur des prémisses erronées ${ }^{91}$. En cela, Crésus confirme la lecture que nous avons faite de la double consultation. Il est le contre-exemple barbare de ce que les Grecs ne doivent pas faire.

Décidément, les temps sont durs pour nos anciennes certitudes sur la divination grecque. Mais c'est aussi la remise en question des certitudes qui nous mènera à une connaissance plus juste et équilibrée de cette institution fondamentale et si longtemps mise entre parenthèses, car elle touchait l'hellénisme «rationnel» dans ce qui apparaissait aux chercheurs comme un trait foncièrement irrationnel.

Pierre BONNECHERE

Département d'histoire et Centre d'études classiques

CP 6128, succ. Centre-ville

MONTRÉAL (Qc) H3C 3J7

Courriel:pierre.bonnechere@umontreal.ca

91 Fontenrose, o.c. (n. 9), Q 99-103 : en part. Hérodote, I, 46-91. Je dois cette remarque à Giulia Sissa. On verra aussi les oracles rapportés par Xénophon (Cyropédie, 19-20), qui se démarquent un peu de ceux d'Hérodote : Crésus demande au dieu s'il aura des enfants, et quand après de nombreux refus, il obtient une réponse, Apollon l'assure qu'il en aura. Par la suite, ses enfants ne lui apportant aucune satisfaction, il interroge encore Apollon pour savoir comment il sera heureux, et la réponse est, toute classique, de « se connaître lui-même » (Q 104-105). 


\section{Note additionnelle}

Dans le contexte des rêves à répétition, signalons aussi la «chronique du sérapiéion de Délos ». Le $3^{e}$ prêtre se targue d'avoir bâti un temple à Sarapis «en six mois », histoire dans laquelle le dieu intervient à plusieurs reprises, en songe et par signe manifeste, et qui rappelle celle du Zoilos, le serviteur du dioicète Apollonios (ci-dessus 2.1.12). Voir RICIS 202/0101 - ca 250-220 av. J.-C. (IG XI [4] 1299). Voir en dernier lieu : B. DIGNAS, "'Greek' priests of Sarapis? ", in B. Dignas, K. TRAmPEDACH (éd.), Practitioners of the Divine: Greek Priests and Religious Officials from Homer to Heliodorus, Cambridge (MA), 2008, p. $73-88$ (avec bibl.). 